\title{
A rigidity property of some negatively curved solvable Lie groups
}

Nageswari Shanmugalingam and Xiangdong Xie

Abstract. We show that for some negatively curved solvable Lie groups, all self quasiisometries are almost isometries. We prove this by showing that all self quasisymmetric maps of the ideal boundary (of the solvable Lie groups) are biLipschitz with respect to the visual metric.

Mathematics Subject Classification (2010). 20F65, 30C65, 53C20.

Keywords. Quasiisometry, quasisymmetric map, negatively curved solvable Lie groups.

\section{Introduction}

In recent years, there have been a lot of interest in the large scale geometry of solvable Lie groups and finitely generated solvable groups ([D], [EFW1], [EFW2], [FM1], [FM2], [FM3], [Pe]). In particular, Eskin, Fisher and Whyte ([EFW1], [EFW2]) proved the quasiisometric rigidity of the 3-dimensional solvable Lie group Sol. In this paper, we use quasiconformal analysis to prove a rigidity property of some negatively curved solvable Lie groups.

Let $A$ be an $n \times n$ diagonal matrix with real eigenvalues $\alpha_{i}$ with $\alpha_{i+1}>\alpha_{i}>0$ :

$$
A=\left(\begin{array}{cccc}
\alpha_{1} I_{n_{1}} & \mathbf{0} & \ldots & \mathbf{0} \\
\mathbf{0} & \alpha_{2} I_{n_{2}} & \ldots & \mathbf{0} \\
\ldots & \ldots & \ldots & \ldots \\
\mathbf{0} & \mathbf{0} & \ldots & \alpha_{r} I_{n_{r}}
\end{array}\right)
$$

where $I_{n_{i}}$ is the $n_{i} \times n_{i}$ identity matrix and the $\mathbf{0}$ 's are zero matrices (of various sizes). Let $\mathbb{R}$ act on $\mathbb{R}^{n}$ by the linear transformations $e^{t A}(t \in \mathbb{R})$ and we can form the semidirect product $G_{A}=\mathbb{R}^{n} \rtimes_{A} \mathbb{R}$. That is, $G_{A}=\mathbb{R}^{n} \times \mathbb{R}$ as a smooth manifold, and the group operation is given for all $(x, t),(y, s) \in \mathbb{R}^{n} \times \mathbb{R}$ by

$$
(x, t) \cdot(y, s)=\left(x+e^{t A} y, t+s\right) .
$$

The group $G_{A}$ is a simply connected solvable Lie group and is the subject of study in this paper. 
We endow $G_{A}$ with the left invariant metric determined by taking the standard Euclidean metric at the identity of $G_{A} \approx \mathbb{R}^{n} \times \mathbb{R}=\mathbb{R}^{n+1}$. With this metric $G_{A}$ has sectional curvature $-\alpha_{r}^{2} \leq K \leq-\alpha_{1}^{2}$ (and so is Gromov hyperbolic). Hence $G_{A}$ has a well defined ideal boundary $\partial G_{A}$. There is a so-called cone topology on $\overline{G_{A}}=G_{A} \cup \partial G_{A}$, in which $\partial G_{A}$ is homeomorphic to the $n$-dimensional sphere and $\overline{G_{A}}$ is homeomorphic to the closed $(n+1)$-ball in the Euclidean space. For each $x \in \mathbb{R}^{n}$, the map $\gamma_{x}: \mathbb{R} \rightarrow G_{A}, \gamma_{x}(t)=(x, t)$ is a geodesic. We call such a geodesic a vertical geodesic. It can be checked that all vertical geodesics are asymptotic as $t \rightarrow+\infty$. Hence they define a point $\xi_{0}$ in the ideal boundary $\partial G_{A}$.

Since $G_{A}$ is Gromov hyperbolic, there is a family of visual metrics on $\partial G_{A}$. For each $\xi \in \partial G_{A}$, there is also the so-called parabolic visual metric on $\partial G_{A} \backslash\{\xi\}$. The relation between visual metrics and parabolic visual metrics is analogous to the relation between spherical metric (on the sphere) and the Euclidean metric (on the one point complement of the sphere). We next recall the parabolic visual metric $D$ on $\partial G_{A}$ viewed from $\xi_{0}$.

The set $\partial G_{A} \backslash\left\{\xi_{0}\right\}$ can be naturally identified with $\mathbb{R}^{n}$ (see Section 2). Write $\mathbb{R}^{n}=\mathbb{R}^{n_{1}} \times \cdots \times \mathbb{R}^{n_{r}}$, where $\mathbb{R}^{n_{i}}$ is the eigenspace associated to the eigenvalue $\alpha_{i}$ of $A$. Each point $x \in \mathbb{R}^{n}$ can be written as $x=\left(x_{1}, \ldots, x_{r}\right)$ with $x_{i} \in \mathbb{R}^{n_{i}}$. The parabolic visual metric $D$ on $\partial G_{A} \backslash\left\{\xi_{0}\right\} \approx \mathbb{R}^{n}$ is defined by

$$
D(x, y)=\max \left\{\left|x_{1}-y_{1}\right|,\left|x_{2}-y_{2}\right|^{\alpha_{1} / \alpha_{2}}, \ldots,\left|x_{r}-y_{r}\right|^{\alpha_{1} / \alpha_{r}}\right\},
$$

for all $x=\left(x_{1}, \ldots, x_{r}\right), y=\left(y_{1}, \ldots, y_{r}\right) \in \mathbb{R}^{n}$.

Let $\eta:[0, \infty) \rightarrow[0, \infty)$ be a homeomorphism. An embedding of metric spaces $f: X \rightarrow Y$ is an $\eta$-quasisymmetric embedding if for all distinct triples $x, y, z \in X$, we have

$$
\frac{d(f(x), f(y))}{d(f(x), f(z))} \leq \eta\left(\frac{d(x, y)}{d(x, z)}\right) .
$$

If $f$ is further assumed to be a homeomorphism, we say it is $\eta$-quasisymmetric. A map $f: X \rightarrow Y$ is quasisymmetric if it is $\eta$-quasisymmetric for some $\eta$.

When $r \geq 2$, Bruce Kleiner has proved that ([K]) every self quasisymmetry of $\partial G_{A}$ (equipped with a visual metric) preserves the horizontal foliation (see Section 3) and fixes the point $\xi_{0}$. This is one of the main ingredients in the proof of our main result. Since Kleiner's proof is unpublished, we include a proof here for completeness. Notice that Kleiner's result implies that a self quasisymmetry of $\partial G_{A}$ induces a self map of $\left(\mathbb{R}^{n}, D\right)$.

The following is the main result of this paper.

Theorem 1.1. Let $G_{A}$ and $\xi_{0} \in \partial G_{A}$ be as above. If $r \geq 2$, then every self quasisymmetry of $\partial G_{A}$ (equipped with a visual metric) is biLipschitz on $\partial G_{A} \backslash\left\{\xi_{0}\right\}$ with respect to the parabolic visual metric $D$. 
One should compare this with quasiconformal maps on Euclidean spaces ([GV]) and Heisenberg groups ([B]), where there are non-biLipschitz quasiconformal maps. On the other hand, the conclusion of Theorem 1.1 is not as strong as in the cases of quaternionic hyperbolic spaces, Cayley plane ([P]) and Fuchsian buildings ([BP], $[\mathrm{X}]$ ), where every quasisymmetric map of the ideal boundary is actually a conformal map. In our case, there are many non-conformal quasisymmetric maps of the ideal boundary of $G_{A}$. We also remark that in Section 15 of [T2] Tyson has previously classified (quasi)metric spaces of the form $\left(\mathbb{R}^{n}, D\right)$ up to quasisymmetry.

We list three consequences of Theorem 1.1.

Let $L \geq 1$ and $C \geq 0$. A (not necessarily continuous ) map $f: X \rightarrow Y$ between two metric spaces is an $(L, A)$-quasiisometry if

(1) $d\left(x_{1}, x_{2}\right) / L-C \leq d\left(f\left(x_{1}\right), f\left(x_{2}\right)\right) \leq L d\left(x_{1}, x_{2}\right)+C$ for all $x_{1}, x_{2} \in X$;

(2) for any $y \in Y$, there is some $x \in X$ with $d(f(x), y) \leq C$.

In the case $L=1$, we call $f$ an almost isometry.

Corollary 1.2. Assume that $r \geq 2$. Then every self quasiisometry of $G_{A}$ is an almost isometry.

Notice that an almost isometry is not necessarily a finite distance away from an isometry.

The following result was previously obtained by B. Kleiner [K].

Corollary 1.3. If $r \geq 2$, then $G_{A}$ is not quasiisometric to any finitely generated group.

In the identification of $G_{A}$ with $\mathbb{R}^{n} \times \mathbb{R}$, we view the map $h: \mathbb{R}^{n} \times \mathbb{R} \rightarrow \mathbb{R}$, $h(x, t)=t$ as the height function. A quasiisometry $\varphi$ of $G_{A}$ is height-respecting if $|h(\varphi(x, t))-t|$ is bounded independent of $x, t$.

Corollary 1.4. Assume that $r \geq 2$. Then all self quasiisometries of $G_{A}$ are heightrespecting.

The question of whether a quasiisometry of $G_{A}$ is height-respecting is important for the following three reasons. First, Farb and Mosher ([FM1]) have classified a large class of solvable Lie groups (including groups of type $G_{A}$ ) up to heightrespecting quasiisometries. Second, there is no known examples of non-heightrespecting quasiisometries except for rank one symmetric spaces of noncompact type. Finally, showing a quasiisometry is height-respecting is a key step in the proof of the quasiisometric rigidity of Sol ([EFW1], [EFW2]).

When $r=1$, the group $G_{A}$ is isometric to a rescaling of the real hyperbolic space. In this case, all the above results fail. 
This paper is structured as follows. In Section 2 we review some basics about the group $G_{A}$. In Section 3 we prove that quasisymmetric self-maps of $\partial G_{A} \backslash\left\{\xi_{0}\right\}$ equipped with the parabolic visual metric preserve horizontal foliations, and in Section 4 we will prove that such maps are biLipschitz with respect to this metric. In Section 5 we provide the proofs of Theorem 1.1 and the corollaries stated in Section 1.

Acknowledgment. We would like to thank Bruce Kleiner for helpful discussions.

\section{The solvable Lie groups $G_{A}$}

In this section we review some basic facts about the group $G_{A}$ and define several parabolic visual (quasi)metrics on the ideal boundary.

Let $A$ and $G_{A}$ be as in the Introduction. We endow $G_{A}$ with the left invariant metric determined by taking the standard Euclidean metric at the identity of $G_{A} \approx$ $\mathbb{R}^{n} \times \mathbb{R}=\mathbb{R}^{n+1}$. At a point $(x, t) \in \mathbb{R}^{n} \times \mathbb{R} \approx G_{A}$, the tangent space is identified with $\mathbb{R}^{n} \times \mathbb{R}$, and the Riemannian metric is given by the symmetric matrix

$$
\left(\begin{array}{cc}
e^{-2 t A} & 0 \\
0 & 1
\end{array}\right)
$$

With this metric $G_{A}$ has sectional curvature $-\alpha_{r}^{2} \leq K \leq-\alpha_{1}^{2}$. Hence $G_{A}$ has a well defined ideal boundary $\partial G_{A}$. All vertical geodesics $\gamma_{x}\left(x \in \mathbb{R}^{n}\right)$ are asymptotic as $t \rightarrow+\infty$. Hence they define a point $\xi_{0}$ in the ideal boundary $\partial G_{A}$.

The sets $\mathbb{R}^{n} \times\{t\}(t \in \mathbb{R})$ are horospheres centered at $\xi_{0}$. For each $t \in \mathbb{R}$, the induced metric on the horosphere $\mathbb{R}^{n} \times\{t\} \subset G_{A}$ is determined by the quadratic form $e^{-2 t A}$. This metric has distance formula $d_{\mathbb{R}^{n} \times\{t\}}((x, t),(y, t))=\left|e^{-t A}(x-y)\right|$. Here $|\cdot|$ denotes the Euclidean norm. The distance between two horospheres, corresponding to $t=t_{1}$ and $t=t_{2}$, is $\left|t_{1}-t_{2}\right|$. It follows that for $\left(x_{1}, t_{1}\right),\left(x_{2}, t_{2}\right) \in G_{A}=\mathbb{R}^{n} \times \mathbb{R}$,

$$
d\left(\left(x_{1}, t_{1}\right),\left(x_{2}, t_{2}\right)\right) \geq\left|t_{1}-t_{2}\right| .
$$

Each geodesic ray in $G_{A}$ is asymptotic to either an upward oriented vertical geodesic or a downward oriented vertical geodesic. The upward oriented geodesics are asymptotic to $\xi_{0}$ and the downward oriented vertical geodesics are in 1-to-1 correspondence with $\mathbb{R}^{n}$. Hence $\partial G_{A} \backslash\left\{\xi_{0}\right\}$ can be naturally identified with $\mathbb{R}^{n}$.

Recall that a quasimetric on a set $A$ is a function $\rho: A \times A \rightarrow[0, \infty)$ satisfying: (1) $\rho(x, y)=\rho(y, x)$ for all $x, y \in A$; (2) $\rho(x, y)=0$ only when $x=y$; (3) there is a constant $L \geq 1$ such that $\rho(x, z) \leq L(\rho(x, y)+\rho(y, z))$ for all $x, y, z \in A$.

Given $x, y \in \mathbb{R}^{n} \approx \partial G_{A} \backslash\left\{\xi_{0}\right\}$, the parabolic visual quasimetric $D_{e}(x, y)$ is defined as follows: $D_{e}(x, y)=e^{t}$, where $t$ is the unique real number such that at height $t$ the two vertical geodesics $\gamma_{x}$ and $\gamma_{y}$ are at distance one apart in the horosphere; that is, $d_{\mathbb{R}^{n} \times\{t\}}((x, t),(y, t))=\left|e^{-t A}(x-y)\right|=1$. Here the subscript $e$ 
in $D_{e}$ means it corresponds to the Euclidean norm. This definition of parabolic visual quasimetric is very natural, but $D_{e}$ does not have a simple formula. Next we describe another parabolic visual quasimetric which is biLipschitz equivalent with $D_{e}$ and admits a simple formula.

In addition to the Euclidean norm, there is another norm on $\mathbb{R}^{n}$ that is naturally associated to $G_{A}$. Write $\mathbb{R}^{n}=\mathbb{R}^{n_{1}} \times \cdots \times \mathbb{R}^{n_{r}}$, where $\mathbb{R}^{n_{i}}$ is the eigenspace associated to the eigenvalue $\alpha_{i}$ of $A$. Each point $x \in \mathbb{R}^{n}$ can be written as $x=\left(x_{1}, \ldots, x_{r}\right)$ with $x_{i} \in \mathbb{R}^{n_{i}}$. The block supernorm is given by $|x|_{s}=\max \left\{\left|x_{1}\right|, \ldots,\left|x_{r}\right|\right\}$ for $x=$ $\left(x_{1}, \ldots, x_{r}\right)$. Using this norm one can define another parabolic visual quasimetric on $\partial G_{A} \backslash\left\{\xi_{0}\right\}$ as follows: $D_{s}(x, y)=e^{t}$, where $t$ is the unique real number such that at height $t$ the two vertical geodesics $\gamma_{x}$ and $\gamma_{y}$ are at distance one apart with respect to the norm $|\cdot|_{s}$; that is, $\left|e^{-t A}(x-y)\right|_{s}=1$. Here the subscript $s$ in $D_{s}$ means it corresponds to the block supernorm $|\cdot|_{s}$. Then $D_{s}$ is given by Lemma 7 of [D]:

$$
D_{s}(x, y)=\max \left\{\left|x_{1}-y_{1}\right|^{\frac{1}{\alpha_{1}}}, \ldots,\left|x_{r}-y_{r}\right|^{\frac{1}{\alpha_{r}}}\right\},
$$

for all $x=\left(x_{1}, \ldots, x_{r}\right), y=\left(y_{1}, \ldots, y_{r}\right) \in \mathbb{R}^{n}$.

Notice that $|x|_{s} \leq|x| \leq \sqrt{r}|x|_{s}$ for all $x \in \mathbb{R}^{n}$. Using this, one can verify the following lemma, whose proof is left to the reader.

Lemma 2.1. For all $x, y \in \mathbb{R}^{n}$ we have $D_{s}(x, y) \leq D_{e}(x, y) \leq r^{1 / 2 \alpha_{1}} D_{s}(x, y)$.

In general, $D_{s}$ does not satisfy the triangle inequality. However, for each $0<$ $\epsilon \leq \alpha_{1}$, the function $D_{s}^{\epsilon}$ is always a metric, called a parabolic visual metric. In this paper we consider the following parabolic visual metric

$$
D(x, y)=D_{s}^{\alpha_{1}}(x, y)=\max \left\{\left|x_{1}-y_{1}\right|,\left|x_{2}-y_{2}\right|^{\alpha_{1} / \alpha_{2}}, \ldots,\left|x_{r}-y_{r}\right|^{\alpha_{1} / \alpha_{r}}\right\} .
$$

With respect to this metric the rectifiable curves in $\mathbb{R}^{n} \approx \partial G_{A} \backslash\left\{\xi_{0}\right\}$ are necessarily curves of the form $\gamma: I \rightarrow \mathbb{R}^{n}$ with $\gamma(t)=\left(\gamma_{1}(t), c_{2}, \ldots, c_{r}\right)$ where $c_{i} \in \mathbb{R}^{n_{i}}$, $2 \leq i \leq r$, are constant vectors. This follows from the fact that the directions corresponding to $\mathbb{R}^{n_{i}}, i \geq 2$, have their Euclidean distance components "snowflaked" by the power $\alpha_{1} / \alpha_{i}<1$.

\section{Quasisymmetric maps preserve horizontal foliations}

In this section we show that every self-quasisymmetry of $\left(\mathbb{R}^{n}, D\right)$ preserves a natural foliation on $\mathbb{R}^{n}$. We remark that this fact has been observed previously by both B. Kleiner [K] and J. Tyson (Section 15, [T2]). We include a proof here for completeness.

Recall that a metric space $X$ endowed with a Borel measure $\mu$ is an Ahlfors regular space of dimension $Q$ (for short, a $Q$-regular space) if there exists a constant $C_{0} \geq 1$ 
so that

$$
C_{0}^{-1} r^{Q} \leq \mu\left(B_{r}\right) \leq C_{0} r^{Q}
$$

for every ball $B_{r}$ with radius $r<\operatorname{diam}(X)$.

We need the following result; see [T1] for the definition of the $\operatorname{modulus}_{\operatorname{Mod}}$ of a family of curves.

Theorem 3.1 ([T1], Theorem 1.4). Let $X$ and $Y$ be locally compact, connected, $Q$-regular metric spaces $(Q>1)$ and let $f: X \rightarrow Y$ be an $\eta$-quasisymmetric homeomorphism. Then there is a constant $C$ depending only on $\eta, Q$ and the regularity constants of $X$ and $Y$ so that

$$
\frac{1}{C} \operatorname{Mod}_{Q} \Gamma \leq \operatorname{Mod}_{Q} f(\Gamma) \leq C \operatorname{Mod}_{Q} \Gamma
$$

for every curve family $\Gamma$ in $X$.

Recall that we write $\mathbb{R}^{n}$ as $\mathbb{R}^{n}=\mathbb{R}^{n_{1}} \times \cdots \times \mathbb{R}^{n_{r}}$. Set $Y=\mathbb{R}^{n_{2}} \times \cdots \times \mathbb{R}^{n_{r}}$ and write $\mathbb{R}^{n}=\mathbb{R}^{n_{1}} \times Y$. Since we assume $r \geq 2$, the set $Y$ is nontrivial. The subsets $\left\{\mathbb{R}^{n_{1}} \times\{y\}: y \in Y\right\}$ form a foliation of $\mathbb{R}^{n}$. We call this foliation the horizontal foliation and each leaf $\mathbb{R}^{n_{1}} \times\{y\}$ a horizontal leaf. Since $\frac{\alpha_{1}}{\alpha_{i}}<1$ for all $2 \leq i \leq r$, we notice that a curve in $\left(\mathbb{R}^{n}, D\right)$ is not rectifiable if it is not contained in a horizontal leaf.

Observe that $\left(\mathbb{R}^{n_{i}},|\cdot|^{\alpha_{1} / \alpha_{i}}\right)$ with the Hausdorff measure (which is comparable to the $n_{i}$-dimensional Lebesgue measure) is $n_{i} \alpha_{i} / \alpha_{1}$-regular. Let $\mu$ be the product of the Hausdorff measures on the factors $\left(\mathbb{R}^{n_{i}},|\cdot|^{\alpha_{1} / \alpha_{i}}\right)$. Then it is easy to see that $\left(\mathbb{R}^{n}, D\right)$ with the measure $\mu$ is $Q$-regular with $Q=\sum_{i=1}^{r} n_{i} \frac{\alpha_{i}}{\alpha_{1}}$. It follows that Theorem 3.1 applies to the metric space $\left(\mathbb{R}^{n}, D\right)$. We also point out here that the Hausdorff measure $\mu$ is comparable to the canonical $n$-dimensional Lebesgue measure on $\mathbb{R}^{n}$.

Theorem 3.2. If $r \geq 2$, then every quasisymmetry $F:\left(\mathbb{R}^{n}, D\right) \rightarrow\left(\mathbb{R}^{n}, D\right)$ preserves the horizontal foliation on $\mathbb{R}^{n}$.

Proof. Suppose $F$ does not preserve the horizontal foliation. Then there are two points $p$ and $q$ in some $\mathbb{R}^{n_{1}} \times\{y\}$ such that $f(p)$ and $f(q)$ are not in the same horizontal leaf. Let $\gamma$ be the Euclidean line segment from $p$ to $q$ and $\Gamma$ be the family of straight segments parallel to $\gamma$ in $\mathbb{R}^{n}$ whose union is an $n$-dimensional circular cylinder with $\gamma$ as the central axis. The curves in $\Gamma$ are rectifiable with respect to the metric $D$. Since $f$ is a homeomorphism, by choosing the radius of the circular cylinder to be sufficiently small (by a compactness argument) we may assume that no curve in $\Gamma$ is mapped into a horizontal leaf. It follows that $f(\Gamma)$ has no locally rectifiable curve and so $\operatorname{Mod}_{Q} f(\Gamma)=0$. On the other hand, [V1], 7.2 (page 21), shows that $\operatorname{Mod}_{Q} \Gamma>0$ (the Euclidean length element on each $\beta \in \Gamma$ is the same 
as the length element on $\beta$ obtained from the metric $D$ ). Since $Q=\Sigma_{i=1}^{r} n_{i} \frac{\alpha_{i}}{\alpha_{1}}>1$, this contradicts Theorem 3.1. Hence each horizontal leaf is mapped to a horizontal leaf.

\section{Quasisymmetry implies biLipschitz}

In this section we show that each self quasisymmetry of $\left(\mathbb{R}^{n}, D\right)$ is actually a biLipschitz map. One should contrast this with the case of Euclidean spaces and Heisenberg groups, where there are non-biLipschitz quasisymmetric maps ([GV], $[\mathrm{B}])$. On the other hand, $\left(\mathbb{R}^{n}, D\right)$ is not as rigid as the ideal boundary of a quaternionic hyperbolic space or a Cayley plane ([P]) or a Fuchsian building ([BP], $[\mathrm{X}])$, where each self quasisymmetry is a conformal map.

Let $K \geq 1$ and $C>0$. A bijection $F: X_{1} \rightarrow X_{2}$ between two metric spaces is called a $K$-quasisimilarity (with constant $C$ ) if

$$
\frac{C}{K} d(x, y) \leq d(F(x), F(y)) \leq C K d(x, y)
$$

for all $x, y \in X_{1}$. It is clear that a map is a quasisimilarity if and only if it is a biLipschitz map. The point of using the notion of quasisimilarity is that sometimes there is control on $K$ but not on $C$.

Theorem 4.1. Let $F:\left(\mathbb{R}^{n}, D\right) \rightarrow\left(\mathbb{R}^{n}, D\right)$ be an $\eta$-quasisymmetry. Then $F$ is a $K$-quasisimilarity with $K=\left(\eta(1) / \eta^{-1}(1)\right)^{2 r+2}$.

In this section, we first develop some intermediate results, and then use these results to provide a proof of this theorem. We first recall some definitions.

Let $g: X_{1} \rightarrow X_{2}$ be a homeomorphism between two metric spaces. We define for every $x \in X_{1}$ and $r>0$,

$$
\begin{aligned}
L_{g}(x, r) & =\sup \left\{d\left(g(x), g\left(x^{\prime}\right)\right): d\left(x, x^{\prime}\right) \leq r\right\}, \\
l_{g}(x, r) & =\inf \left\{d\left(g(x), g\left(x^{\prime}\right)\right): d\left(x, x^{\prime}\right) \geq r\right\} .
\end{aligned}
$$

Notice that if $X_{1}$ is connected and $X_{1} \backslash B(x, r)$ is non-empty, then $l_{g}(x, r) \leq$ $L_{g}(x, r)$. In this paper, we only consider connected metric spaces. Set

$$
L_{g}(x)=\limsup _{r \rightarrow 0} \frac{L_{g}(x, r)}{r}, \quad l_{g}(x)=\liminf _{r \rightarrow 0} \frac{l_{g}(x, r)}{r} .
$$

Then

$$
L_{g^{-1}}(g(x))=\frac{1}{l_{g}(x)} \quad \text { and } \quad l_{g^{-1}}(g(x))=\frac{1}{L_{g}(x)}
$$


for any $x \in X_{1}$. If $g$ is an $\eta$-quasisymmetry, then $L_{g}(x, r) \leq \eta(1) l_{g}(x, r)$ for all $x \in X_{1}$ and $r>0$. Hence if in addition

$$
\lim _{r \rightarrow 0} \frac{L_{g}(x, r)}{r} \text { or } \quad \lim _{r \rightarrow 0} \frac{l_{g}(x, r)}{r}
$$

exist, then

$$
0 \leq l_{g}(x) \leq L_{g}(x) \leq \eta(1) l_{g}(x) \leq \infty .
$$

Recall the decomposition $\mathbb{R}^{n}=\mathbb{R}^{n_{1}} \times Y$. Given points $y=\left(x_{2}, \ldots, x_{r}\right)$ and $y^{\prime}=\left(x_{2}^{\prime}, \ldots, x_{r}^{\prime}\right) \in Y$ with $x_{i}, x_{i}^{\prime} \in \mathbb{R}^{n_{i}}$, set

$$
D_{Y}\left(y, y^{\prime}\right)=\max \left\{\left|x_{2}-x_{2}^{\prime}\right|^{\frac{\alpha_{1}}{\alpha_{2}}},\left|x_{3}-x_{3}^{\prime}\right|^{\frac{\alpha_{1}}{\alpha_{3}}}, \ldots,\left|x_{r}-x_{r}^{\prime}\right|^{\frac{\alpha_{1}}{\alpha_{r}}}\right\} .
$$

For $p=\left(x_{1}, y\right), p^{\prime}=\left(x_{1}^{\prime}, y^{\prime}\right) \in \mathbb{R}^{n_{1}} \times Y$, we have $D\left(p, p^{\prime}\right)=\max \left\{\mid x_{1}-\right.$ $\left.x_{1}^{\prime} \mid, D_{Y}\left(y, y^{\prime}\right)\right\}$. We notice that for every $y_{1}, y_{2} \in Y$, the distance in the metric $D$ of the two horizontal leaves,

$$
D\left(\mathbb{R}^{n_{1}} \times\left\{y_{1}\right\}, \mathbb{R}^{n_{1}} \times\left\{y_{2}\right\}\right)=D_{Y}\left(y_{1}, y_{2}\right) .
$$

Also, for any $p=\left(x_{1}, y_{1}\right) \in \mathbb{R}^{n_{1}} \times Y$ and any $y_{2} \in Y$,

$$
D\left(p, \mathbb{R}^{n_{1}} \times\left\{y_{2}\right\}\right)=D_{Y}\left(y_{1}, y_{2}\right) .
$$

By Theorem 3.2 the quasisymmetry $F$ preserves the horizontal foliation. Hence it induces a map $G: Y \rightarrow Y$ such that for any $y \in Y, F\left(\mathbb{R}^{n_{1}} \times\{y\}\right)=\mathbb{R}^{n_{1}} \times$ $\{G(y)\}$. For each $y \in Y$, let $H(\cdot, y): \mathbb{R}^{n_{1}} \rightarrow \mathbb{R}^{n_{1}}$ be the map such that $F(x, y)=$ $(H(x, y), G(y))$ for all $x \in \mathbb{R}^{n_{1}}$. Because $F:\left(\mathbb{R}^{n}, D\right) \rightarrow\left(\mathbb{R}^{n}, D\right)$ is an $\eta$ quasisymmetry, it follows that for each fixed $y \in Y$, the map $H(\cdot, y): \mathbb{R}^{n_{1}} \rightarrow \mathbb{R}^{n_{1}}$ is an $\eta$-quasisymmetry with respect to the Euclidean metric on $\mathbb{R}^{n_{1}}$. The following lemma together with equations (4.1) and (4.2) imply that $G:\left(Y, D_{Y}\right) \rightarrow\left(Y, D_{Y}\right)$ is also an $\eta$-quasisymmetry.

Lemma 4.2 ([T2], Lemma 15.9). Let $g: X_{1} \rightarrow X_{2}$ be an $\eta$-quasisymmetry and $A, B, C \subset X_{1}$. If $d(A, B) \leq t d(A, C)$ for some $t \geq 0$, then there is some $a \in A$ such that

$$
d(g(A), g(B)) \leq \eta(t) d(g(a), g(C)) .
$$

We recall that if $g: X_{1} \rightarrow X_{2}$ is an $\eta$-quasisymmetry, then $g^{-1}: X_{2} \rightarrow X_{1}$ is an $\eta_{2}$-quasisymmetry, where $\eta_{2}(t)=\left(\eta^{-1}\left(t^{-1}\right)\right)^{-1}$, see [V2], Theorem 6.3. Note that $\eta_{2}(1)=1 / \eta^{-1}(1)$ and $\eta_{2}^{-1}(1)=1 / \eta(1)$.

In the proofs of the following lemmas, the quantities $l_{G}, L_{G}, l_{G^{-1}}, L_{G^{-1}}$ are defined with respect to the metric $D_{Y}$. Similarly, $l_{H(\cdot, y)}, L_{H(\cdot, y)}, l_{I_{y}}$ and $L_{I_{y}}$ are defined with respect to the Euclidean metric on $\mathbb{R}^{n_{1}}$, where $I_{y}:=H(\cdot, y)^{-1}: \mathbb{R}^{n_{1}} \rightarrow$ $\mathbb{R}^{n_{1}}$. Lemmas 4.6 and 4.7 together verify Theorem 4.1 for the case $r=2$. At the end of this section we will use induction to then complete the proof of Theorem 4.1 for the general case $r \geq 2$. 
Lemma 4.3. The following holds for all $y \in Y$ and $x \in \mathbb{R}^{n_{1}}$ :

(1) $L_{G}(y, r) \leq \eta(1) l_{H(\cdot, y)}(x, r)$ for $r>0$;

(2) $\eta^{-1}(1) l_{H(\cdot, y)}(x) \leq l_{G}(y) \leq \eta(1) l_{H(\cdot, y)}(x)$;

(3) $\eta^{-1}(1) L_{H(\cdot, y)}(x) \leq L_{G}(y) \leq \eta(1) L_{H(\cdot, y)}(x)$.

Proof. To prove (1), let $y \in Y, x \in \mathbb{R}^{n_{1}}$ and $r>0$. Let $y^{\prime} \in Y$ be an arbitrary point with $D_{Y}\left(y, y^{\prime}\right) \leq r$ and $x^{\prime} \in \mathbb{R}^{n_{1}}$ an arbitrary point with $\left|x-x^{\prime}\right| \geq r$. Then $D\left((x, y),\left(x, y^{\prime}\right)\right) \leq r \leq D\left((x, y),\left(x^{\prime}, y\right)\right)$. Since $F$ is $\eta$-quasisymmetric, we have

$$
\begin{aligned}
D_{Y}\left(G(y), G\left(y^{\prime}\right)\right) \leq D\left(F(x, y), F\left(x, y^{\prime}\right)\right) & \leq \eta(1) D\left(F(x, y), F\left(x^{\prime}, y\right)\right) \\
& =\eta(1)\left|H(x, y)-H\left(x^{\prime}, y\right)\right| .
\end{aligned}
$$

Since $y^{\prime}$ and $x^{\prime}$ are chosen arbitrarily and are independent of each other, the inequality follows.

Next we prove (2) and (3). Since $Y$ is connected, we have $l_{G}(y, r) \leq L_{G}(y, r)$. Now the second inequality of (2) follows from (1). Similarly the second inequality of (3) follows from (1) and the fact that $l_{H(\cdot, y)}(x, r) \leq L_{H(\cdot, y)}(x, r)$.

To prove the first inequalities in (2) and (3), observe that the inverse map

$$
F^{-1}:\left(\mathbb{R}^{n}, D\right) \rightarrow\left(\mathbb{R}^{n}, D\right)
$$

is an $\eta_{2}$-quasisymmetry, with

$$
F^{-1}(x, y)=\left(H\left(\cdot, G^{-1}(y)\right)^{-1}(x), G^{-1}(y)\right)=\left(I_{G^{-1}(y)}(x), G^{-1}(y)\right) .
$$

Applying the second inequality of (2) proven above to $I_{y}$ and $G^{-1}$, we obtain

$$
\frac{1}{L_{G}(y)}=l_{G^{-1}}(G(y)) \leq \eta_{2}(1) \cdot l_{I_{y}}(H(x, y))=\frac{1}{\eta^{-1}(1)} \cdot \frac{1}{L_{H(\cdot, y)}(x)},
$$

hence $L_{G}(y) \geq \eta^{-1}(1) L_{H(\cdot, y)}(x)$, which is the first inequality of (3). Similarly, using the second inequality of (3) we obtain the first inequality of (2).

When $r=2$, we have $Y=\mathbb{R}^{n_{2}}$ and $D_{Y}=|\cdot|^{\frac{\alpha_{1}}{\alpha_{2}}}$.

Lemma 4.4. Assume that $r=2$. Then $0<l_{G}(y) \leq L_{G}(y) \leq \eta(1) l_{G}(y)<\infty$ for a.e. $y \in Y$ with respect to the Lebesgue measure on $Y=\mathbb{R}^{n_{2}}$.

Proof. Observe in this case that $D_{Y}\left(y, y^{\prime}\right)=\left|y-y^{\prime}\right|^{\alpha_{1} / \alpha_{2}}$ for $y, y^{\prime} \in Y=\mathbb{R}^{n_{2}}$. Because $G$ is an $\eta$-quasisymmetry with respect to the metric $D_{Y}$, it is $\eta_{1}$-quasisymmetric with respect to the Euclidean metric, where $\eta_{1}(t)=\left(\eta\left(t^{\alpha_{1} / \alpha_{2}}\right)\right)^{\alpha_{2} / \alpha_{1}}$. Hence the map $G:\left(\mathbb{R}^{n_{2}},|\cdot|\right) \rightarrow\left(\mathbb{R}^{n_{2}},|\cdot|\right)$ is differentiable a.e. with respect to the Lebesgue measure. With $L_{G}^{e}, l_{G}^{e}$ the distortion quantities of the map $G$ with respect to the Euclidean metric, the differentiability property of $G$ shows that $\lim _{r \rightarrow 0} \frac{L_{G}^{e}(y, r)}{r}$ and $\lim _{r \rightarrow 0} \frac{l_{G}^{e}(y, r)}{r}$ 
exist. Since $L_{G}(y, r)=L_{G}^{e}\left(y, r^{\alpha_{2} / \alpha_{1}}\right)^{\alpha_{1} / \alpha_{2}}$ and $l_{G}(y, r)=l_{G}^{e}\left(y, r^{\alpha_{2} / \alpha_{1}}\right)^{\alpha_{1} / \alpha_{2}}$, this implies that both $\lim _{r \rightarrow 0} \frac{L_{G}(y, r)}{r}$ and $\lim _{r \rightarrow 0} \frac{l_{G}(y, r)}{r}$ exist for a.e. $y \in Y$. It follows that

$$
0 \leq l_{G}(y) \leq L_{G}(y) \leq \eta(1) l_{G}(y) \leq \infty .
$$

Fix $y \in Y$ such that both $\lim _{r \rightarrow 0} \frac{L_{G}(y, r)}{r}$ and $\lim _{r \rightarrow 0} \frac{l_{G}(y, r)}{r}$ exist. We next prove that $L_{G}(y) \neq 0, \infty$. Suppose that $L_{G}(y)=\infty$. Then $l_{G}(y)=\infty$ and so by Lemma $4.3(2), l_{H(\cdot, y)}(x)=\infty$ for all $x \in \mathbb{R}^{n_{1}}$. Hence $I_{y}=H(\cdot, y)^{-1}: \mathbb{R}^{n_{1}} \rightarrow$ $\mathbb{R}^{n_{1}}$ has the property that $L_{I_{y}}(x)=0$ for all $x \in \mathbb{R}^{n_{1}}$. This implies that $I_{y}$ is a constant map, contradicting the fact that it is a homeomorphism. Similarly we use Lemma 4.3 (3) to show that $L_{G}(y) \neq 0$.

In the next two lemmas we use the fact that $\eta(1) \geq 1$ and $0<\eta^{-1}(1) \leq 1$.

Lemma 4.5. Suppose that $r=2$. Then, for a.e. $y \in Y$, the map $H(\cdot, y): \mathbb{R}^{n_{1}} \rightarrow \mathbb{R}^{n_{1}}$ is an $\eta(1) / \eta^{-1}(1)$-quasisimilarity with constant $l_{G}(y)>0$.

Proof. By Lemma 4.3 (2) we have $l_{H(\cdot, y)}(x) \geq l_{G}(y) / \eta(1)$. Lemma 4.3 (3) and Lemma 4.4 imply that, for a.e. $y \in Y$, we have $l_{G}(y)>0$ and

$$
L_{H(\cdot, y)}(x) \leq L_{G}(y) / \eta^{-1}(1) \leq\left(\eta(1) / \eta^{-1}(1)\right) l_{G}(y)
$$

for all $x \in \mathbb{R}^{n_{1}}$. Because $\mathbb{R}^{n_{1}}$ is a geodesic space, for a.e. $y \in Y$ the map $H(\cdot, y)$ is an $\eta(1) / \eta^{-1}(1)$-quasisimilarity with constant $l_{G}(y)$.

Lemma 4.6. If $r=2$, then there exists a constant $C>0$ with the following properties:

(1) For each $y \in Y, H(\cdot, y)$ is an $\left(\eta(1) / \eta^{-1}(1)\right)^{4}$-quasisimilarity with constant $C$;

(2) $G:\left(Y, D_{Y}\right) \rightarrow\left(Y, D_{Y}\right)$ is an $\left(\eta(1) / \eta^{-1}(1)\right)^{5}$-quasisimilarity with constant $C$.

Proof. (1) Fix any $y_{0} \in Y$ that satisfies both Lemma 4.4 and Lemma 4.5. Set $C=l_{G}\left(y_{0}\right)$. Let $y \in Y$ be an arbitrary point satisfying both Lemma 4.4 and Lemma 4.5. Fix $x_{0} \in \mathbb{R}^{n_{1}}$ and choose $x \in \mathbb{R}^{n_{1}}$ such that $\left|x-x_{0}\right| \geq D_{Y}\left(y, y_{0}\right)$. Then

$$
D\left(\left(x, y_{0}\right),\left(x_{0}, y\right)\right)=D\left((x, y),\left(x_{0}, y\right)\right)=\left|x-x_{0}\right| \text {. }
$$

By choosing $x$ so that in addition $\left|H\left(x, y_{0}\right)-H\left(x_{0}, y\right)\right|>D_{Y}\left(G\left(y_{0}\right), G(y)\right)$, by the $\eta$-quasisymmetry of $F$ we have

$$
\begin{aligned}
\left|H\left(x, y_{0}\right)-H\left(x_{0}, y\right)\right| & =D\left(F\left(x, y_{0}\right), F\left(x_{0}, y\right)\right) \\
& \leq \eta(1) D\left(F(x, y), F\left(x_{0}, y\right)\right)=\eta(1)\left|H(x, y)-H\left(x_{0}, y\right)\right| .
\end{aligned}
$$

By the choice of $y$ and Lemma 4.5, we have

$$
\left|H(x, y)-H\left(x_{0}, y\right)\right| \leq\left(\eta(1) / \eta^{-1}(1)\right) l_{G}(y)\left|x-x_{0}\right| .
$$


On the other hand,

$$
\begin{aligned}
\left|H\left(x, y_{0}\right)-H\left(x_{0}, y\right)\right| & \geq\left|H\left(x, y_{0}\right)-H\left(x_{0}, y_{0}\right)\right|-\left|H\left(x_{0}, y_{0}\right)-H\left(x_{0}, y\right)\right| \\
& \geq \frac{l_{G}\left(y_{0}\right)}{\eta(1) / \eta^{-1}(1)}\left|x-x_{0}\right|-\left|H\left(x_{0}, y_{0}\right)-H\left(x_{0}, y\right)\right| .
\end{aligned}
$$

Combining the above inequalities and letting $\left|x-x_{0}\right| \rightarrow \infty$, we obtain

$$
l_{G}(y) \geq \frac{1}{(\eta(1))^{3}\left(\eta^{-1}(1)\right)^{-2}} l_{G}\left(y_{0}\right)=\frac{C}{(\eta(1))^{3}\left(\eta^{-1}(1)\right)^{-2}} .
$$

Switching the roles of $y_{0}$ and $y$, we obtain $l_{G}(y) \leq(\eta(1))^{3}\left(\eta^{-1}(1)\right)^{-2} l_{G}\left(y_{0}\right)$. By Lemma 4.4, we have

$$
L_{G}(y) \leq \eta(1) l_{G}(y) \leq(\eta(1))^{4}\left(\eta^{-1}(1)\right)^{-2} C .
$$

Because $\mathbb{R}^{n_{1}}$ is a geodesic space, to show that $H(\cdot, y)$ is a quasisimilarity it suffices to gain control over $l_{H(\cdot, y)}$ and $L_{H(\cdot, y)}$. By (4.4) and Lemma $4.3(3)$,

$$
L_{H(\cdot, y)}(x) \leq L_{G}(y) / \eta^{-1}(1) \leq C(\eta(1))^{4}\left(\eta^{-1}(1)\right)^{-3}
$$

for all $x \in \mathbb{R}^{n_{1}}$, and by (4.3) and Lemma 4.3 (2),

$$
l_{H(\cdot, y)}(x) \geq \frac{1}{\eta(1)} l_{G}(y) \geq \frac{C}{(\eta(1))^{4}\left(\eta^{-1}(1)\right)^{-2}} .
$$

for all $x \in \mathbb{R}^{n_{1}}$. Hence for a.e. $y, H(\cdot, y)$ is an $\left(\eta(1) / \eta^{-1}(1)\right)^{4}$-quasisimilarity with constant $C$. A limiting argument shows this is true for all $y$. Hence (1) holds.

(2) Recall that when $r=2$ we have $Y=\mathbb{R}^{n_{2}}$ and $D_{Y}=|\cdot|^{\alpha_{1} / \alpha_{2}}$. Hence to prove (2) it suffices to show that $G:\left(\mathbb{R}^{n_{2}},|\cdot|\right) \rightarrow\left(\mathbb{R}^{n_{2}},|\cdot|\right)$ is a $K$-quasisimilarity with $K=\left(\eta(1) / \eta^{-1}(1)\right)^{5 \alpha_{2} / \alpha_{1}}$. As observed before, $G$ is $\eta_{1}$-quasisymmetric with respect to the Euclidean metric, where $\eta_{1}(t)=\left(\eta\left(t^{\alpha_{1} / \alpha_{2}}\right)\right)^{\alpha_{2} / \alpha_{1}}$. Because $\mathbb{R}^{n_{2}}$ is a geodesic space, it suffices to gain control over $l_{G}^{e}$ and $L_{G}^{e}$, where $l_{G}^{e}$ and $L_{G}^{e}$ are similar to $l_{G}$ and $L_{G}$, but with the Euclidean metric instead of the metric $D_{Y}$. Because $l_{G}^{e}(p)=l_{G}(p)^{\alpha_{2} / \alpha_{1}}$ and $L_{G}^{e}(p)=L_{G}(p)^{\alpha_{2} / \alpha_{1}}$, it suffices to gain control over the quantities $l_{G}$ and $L_{G}$ in terms of $\left(\eta(1) / \eta^{-1}(1)\right)^{5}$.

Notice that (1) implies

$$
\frac{C}{\left(\eta(1) / \eta^{-1}(1)\right)^{4}} \leq l_{H(\cdot, y)}(x) \leq L_{H(\cdot, y)}(x) \leq C\left(\eta(1) / \eta^{-1}(1)\right)^{4}
$$

for all $x \in \mathbb{R}^{n_{1}}$ and all $y \in Y$. By Lemma 4.3, for all $y \in Y$ we have

$$
\frac{C}{\left(\eta(1) / \eta^{-1}(1)\right)^{5}} \leq l_{G}(y) \leq L_{G}(y) \leq C\left(\eta(1) / \eta^{-1}(1)\right)^{5} .
$$

Hence (2) holds. 
Lemma 4.7. Suppose that $r \geq 2$ and there are constants $K \geq 1$ and $C>0$ with the following properties:

(1) $G:\left(Y, D_{Y}\right) \rightarrow\left(Y, D_{Y}\right)$ is a $K$-quasisimilarity with constant $C$;

(2) For each $y \in Y, H(\cdot, y)$ is a $K$-quasisimilarity with constant $C$.

Then $F$ is an $\left(\eta(1) / \eta^{-1}(1)\right) K$-quasisimilarity with constant $C$.

Proof. Let $\left(x_{1}, y_{1}\right),\left(x_{2}, y_{2}\right) \in \mathbb{R}^{n_{1}} \times Y$. We shall first establish a lower bound for $D\left(F\left(x_{1}, y_{1}\right), F\left(x_{2}, y_{2}\right)\right)$. If $\left|x_{1}-x_{2}\right| \leq D_{Y}\left(y_{1}, y_{2}\right)$, then $D\left(\left(x_{1}, y_{1}\right),\left(x_{2}, y_{2}\right)\right)=$ $D_{Y}\left(y_{1}, y_{2}\right)$ and by (1),

$$
\begin{aligned}
D\left(F\left(x_{1}, y_{1}\right), F\left(x_{2}, y_{2}\right)\right) & \geq D_{Y}\left(G\left(y_{1}\right), G\left(y_{2}\right)\right) \\
& \geq \frac{C}{K} D_{Y}\left(y_{1}, y_{2}\right)=\frac{C}{K} D\left(\left(x_{1}, y_{1}\right),\left(x_{2}, y_{2}\right)\right) .
\end{aligned}
$$

If $\left|x_{1}-x_{2}\right|>D_{Y}\left(y_{1}, y_{2}\right)$, then

$$
D\left(\left(x_{1}, y_{1}\right),\left(x_{2}, y_{2}\right)\right)=D\left(\left(x_{1}, y_{2}\right),\left(x_{2}, y_{2}\right)\right)=\left|x_{1}-x_{2}\right|,
$$

and since $F$ is an $\eta$-quasisymmetry, by using (2),

$$
\begin{aligned}
D\left(F\left(x_{1}, y_{1}\right), F\left(x_{2}, y_{2}\right)\right) & \geq \frac{1}{\eta(1)} D\left(F\left(x_{1}, y_{2}\right), F\left(x_{2}, y_{2}\right)\right) \\
& =\frac{1}{\eta(1)}\left|H\left(x_{1}, y_{2}\right)-H\left(x_{2}, y_{2}\right)\right| \\
& \geq \frac{C}{\eta(1) K}\left|x_{1}-x_{2}\right| \\
& =\frac{C}{\eta(1) K} D\left(\left(x_{1}, y_{1}\right),\left(x_{2}, y_{2}\right)\right) .
\end{aligned}
$$

Hence we have a lower bound for $D\left(F\left(x_{1}, y_{1}\right), F\left(x_{2}, y_{2}\right)\right)$.

By (1), $G^{-1}:\left(Y, D_{Y}\right) \rightarrow\left(Y, D_{Y}\right)$ is a $K$-quasisimilarity with constant $C^{-1}$. Similarly, (2) implies that for each $y \in Y,(H(\cdot, y))^{-1}$ is a $K$-quasisimilarity with constant $C^{-1}$. Also recall that $F^{-1}$ is an $\eta_{2}$-quasisymmetry and $F$ is an $\eta$ quasisymmetry. Now the argument in the previous paragraph applied to $F^{-1}$ implies

$$
D\left(F^{-1}\left(x_{1}, y_{1}\right), F^{-1}\left(x_{2}, y_{2}\right)\right) \geq \frac{1}{C K \eta_{2}(1)} D\left(\left(x_{1}, y_{1}\right),\left(x_{2}, y_{2}\right)\right) .
$$

It follows that

$$
\begin{aligned}
D\left(F\left(x_{1}, y_{1}\right), F\left(x_{2}, y_{2}\right)\right) & \leq C K \eta_{2}(1) D\left(\left(x_{1}, y_{1}\right),\left(x_{2}, y_{2}\right)\right) \\
& =\frac{C K}{\eta^{-1}(1)} D\left(\left(x_{1}, y_{1}\right),\left(x_{2}, y_{2}\right)\right)
\end{aligned}
$$

for all $\left(x_{1}, y_{1}\right),\left(x_{2}, y_{2}\right) \in \mathbb{R}^{n}$, completing the proof. 
Proof of Theorem 4.1. We induct on $r$. Lemmas 4.6 and 4.7 yield the desired result in the case $r=2$. Now we assume that $r \geq 3$ and that the Theorem is true for $r-1$. By Lemma 4.2, $F$ induces an $\eta$-quasisymmetry $G:\left(Y, D_{Y}\right) \rightarrow\left(Y, D_{Y}\right)$. It follows that $G$ is $\eta_{1}$-quasisymmetric with respect to the metric $D_{Y}^{\alpha_{2} / \alpha_{1}}$ (and it is easy to verify that this is indeed a metric), where $\eta_{1}(t)=\left[\eta\left(t^{\alpha_{1} / \alpha_{2}}\right)\right]^{\alpha_{2} / \alpha_{1}}$. We point out here that for $\left(x_{2}, \ldots, x_{r}\right),\left(x_{2}^{\prime}, \ldots, x_{r}^{\prime}\right) \in Y$,

$$
\begin{aligned}
& D_{Y}\left(\left(x_{2}, \ldots, x_{r}\right),\left(x_{2}^{\prime}, \ldots, x_{r}^{\prime}\right)\right)^{\alpha_{2} / \alpha_{1}} \\
& \quad=\max \left\{\left|x_{2}-x_{2}^{\prime}\right|,\left|x_{3}-x_{3}^{\prime}\right|^{\alpha_{2} / \alpha_{3}}, \ldots,\left|x_{r}-x_{r}^{\prime}\right|^{\alpha_{2} / \alpha_{r}}\right\} .
\end{aligned}
$$

Hence the induction hypothesis applied to $G:\left(Y, D_{Y}^{\alpha_{2} / \alpha_{1}}\right) \rightarrow\left(Y, D_{Y}^{\alpha_{2} / \alpha_{1}}\right)$ shows that $G$ is an $\left(\eta_{1}(1) / \eta_{1}^{-1}(1)\right)^{2 r}$-quasisimilarity with constant $C$. Therefore $G:\left(Y, D_{Y}\right) \rightarrow$ $\left(Y, D_{Y}\right)$ is a $K_{1}$-quasisimilarity with constant $C^{\alpha_{1} / \alpha_{2}}$, where

$$
K_{1}=\left(\frac{\eta_{1}(1)}{\eta_{1}^{-1}(1)}\right)^{\frac{2 r \alpha_{1}}{\alpha_{2}}}=\left(\frac{\eta(1)}{\eta^{-1}(1)}\right)^{2 r} .
$$

This implies that $C^{\alpha_{1} / \alpha_{2}} / K_{1} \leq l_{G}(y) \leq L_{G}(y) \leq C^{\alpha_{1} / \alpha_{2}} K_{1}$ for all $y \in Y$. Now Lemma 4.3 yields

$$
C^{\alpha_{1} / \alpha_{2}} \frac{1}{K_{1} \eta(1)} \leq l_{H(\cdot, y)}(x) \leq L_{H(\cdot, y)}(x) \leq C^{\alpha_{1} / \alpha_{2}} \frac{K_{1}}{\eta^{-1}(1)}
$$

for all $y \in Y$ and all $x \in \mathbb{R}^{n_{1}}$. Since $\mathbb{R}^{n_{1}}$ is a geodesic space, for each $y \in Y$ the map $H(\cdot, y)$ is a $K_{1} \frac{\eta(1)}{\eta^{-1}(1)}$-quasisimilarity with constant $C^{\alpha_{1} / \alpha_{2}}$. By Lemma 4.7, the map $F$ is a $K_{1}\left(\frac{\eta(1)}{\eta^{-1}(1)}\right)^{2}$-quasisimilarity with constant $C^{\alpha_{1} / \alpha_{2}}$. Here $K_{1}$ is as in (4.5).

\section{Proof of the main results}

In this section we will prove Theorem 1.1 and the corollaries from the introduction.

Let $X$ be a CAT(-1) space. Bourdon [Bo] defined a visual metric $d_{x}$ on $\partial X$ for each $x \in X$. For each $\omega \in \partial X$, there is also a so-called parabolic visual metric on $\partial X \backslash\{\omega\}$, whose definition is recalled below. Let $\omega \in \partial X$, and $b: X \rightarrow \mathbb{R}$ a Busemann function associated to $\omega$. Define the Gromov product with respect to this Busemann function by

$$
\left(x \mid x^{\prime}\right)_{b}=\frac{1}{2}\left(b(x)+b\left(x^{\prime}\right)-d\left(x, x^{\prime}\right)\right),
$$

which extends to points at infinity. The corresponding Hamenstädt metric $[\mathrm{H}] \rho_{b}$ is then defined as $\rho_{b}(\xi, \eta)=e^{-(\xi \mid \eta)_{b}}$ for $\xi, \eta \in \partial X \backslash\{\omega\}$. If $x \in X$ is such that 
$b(x)=0$, then

$$
\rho_{b}(\xi, \eta)=\frac{d_{x}(\xi, \eta)}{d_{x}(\xi, \omega) d_{x}(\eta, \omega)}
$$

for $\xi, \eta \in \partial X \backslash\{\omega\}$, see [FS], Section 2.3.

We remark that parabolic visual metrics have also been considered by HersonskyPaulin [HP] and Bonk-Kleiner [BK].

Let $A$ and $G_{A}$ be as in the introduction, and $g_{0}$ the Riemannian metric on $G_{A}$. Let $g=\alpha_{1}^{2} g_{0}$. Since $\left(G_{A}, g_{0}\right)$ has sectional curvature $-\alpha_{r}^{2} \leq K \leq-\alpha_{1}^{2}$, we conclude that $\left(G_{A}, g\right)$ has sectional curvature $-\alpha_{r}^{2} / \alpha_{1}^{2} \leq K \leq-1$ and hence is $\operatorname{CAT}(-1)$. Notice that $b:=-\alpha_{1} h$ is a Busemann function on $\left(G_{A}, g\right)$ associated to $\xi_{0}$, where $h: G_{A} \rightarrow \mathbb{R}, h(x, t)=t$ is the height function. It is not difficulty to see that for $X=\left(G_{A}, g\right)$, the metric $\rho_{b}$ on $\partial G_{A} \backslash\left\{\xi_{0}\right\}$ is biLipschitz equivalent to $D$.

Proof of Theorem 1.1. Let $F:\left(\partial G_{A}, d_{x}\right) \rightarrow\left(\partial G_{A}, d_{x}\right)$ be a quasisymmetric map, where $d_{x}$ is the Bourdon metric associated to some base point $x \in G_{A}$ in the CAT $(-1)$ space $\left(G_{A}, \alpha_{1}^{2} g_{0}\right)$. We first prove that $F\left(\xi_{0}\right)=\xi_{0}$. Let $D$ be the metric on $\partial G_{A} \backslash\left\{\xi_{0}\right\}=\mathbb{R}^{n}$ considered in the previous sections. We have observed that $D$ is biLipschitz equivalent with the Hamenstädt metric $\rho_{b}$, where $b=-\alpha_{1} h$. Let $\mu$ be the product of the Hausdorff measures on the factors $\left(\mathbb{R}^{n_{i}},|\cdot|^{\frac{\alpha_{1}}{\alpha_{i}}}\right)$ of $\mathbb{R}^{n}$. Then the metric measure space $\left(\mathbb{R}^{n}, D, \mu\right)$ is $Q$-regular with $Q=\Sigma_{i=1}^{r} n_{i} \frac{\alpha_{i}}{\alpha_{1}}$. On $\partial X$ we define a measure $\mu^{\prime}$ as follows: $\mu^{\prime}\left(\left\{\xi_{0}\right\}\right)=0$, and on $\partial G_{A} \backslash\left\{\xi_{0}\right\}=\mathbb{R}^{n}, \mu^{\prime}$ is absolutely continuous with respect to $\mu$ with Radon-Nikodym derivative

$$
\frac{d \mu^{\prime}}{d \mu}(\xi)=\frac{1}{(1+D(o, \xi))^{2 Q}}
$$

for $\xi \in \partial G_{A}$. It can be shown that $\left(\partial G_{A}, d_{x}\right)$ with $\mu^{\prime}$ is also $Q$-regular. Hence Theorem 3.1 applies to the map $F:\left(\partial G_{A}, d_{x}\right) \rightarrow\left(\partial G_{A}, d_{x}\right)$ and the measure $\mu^{\prime}$.

Suppose $F\left(\xi_{0}\right) \neq \xi_{0}$. Then $F^{-1}\left(\xi_{0}\right)$ lies in exactly one horizontal leaf. Fix some $y \in Y$ such that $\mathbb{R}^{n_{1}} \times\{y\}$ does not contain $F^{-1}\left(\xi_{0}\right)$. Notice that the subset $\left(\mathbb{R}^{n_{1}} \times\{y\}\right) \cup\left\{\xi_{0}\right\}$ of $\partial G_{A}$ is an $n_{1}$-dimensional topological sphere. So $F\left(\mathbb{R}^{n_{1}} \times\right.$ $\left.\{y\} \cup\left\{\xi_{0}\right\}\right)$ is an $n_{1}$-dimensional topological sphere in $\mathbb{R}^{n}$. Since each horizontal leaf is an $n_{1}$-dimensional Euclidean space, the set $F\left(\mathbb{R}^{n_{1}} \times\{y\} \cup\left\{\xi_{0}\right\}\right)$ is not contained in any horizontal leaf. It follows that as a dense subset of $F\left(\mathbb{R}^{n_{1}} \times\{y\} \cup\left\{\xi_{0}\right\}\right)$, the set $F\left(\mathbb{R}^{n_{1}} \times\{y\}\right)$ is also not contained in any horizontal leaf. Hence there are two points $p$ and $q$ in $\mathbb{R}^{n_{1}} \times\{y\}$ such that $F(p)$ and $F(q)$ are not in the same horizontal leaf.

Let $\gamma$ be the Euclidean line segment from $p$ to $q$ and $\Gamma$ be the family of straight segments parallel to $\gamma$ in $\mathbb{R}^{n}$ whose union is an $n$-dimensional circular cylinder $C$ with $\gamma$ as the central axis. The curves in $\Gamma$ are rectifiable with respect to the metric $D$. Since $F$ is a homeomorphism, by choosing the radius of the circular cylinder to be sufficiently small (by a compactness argument) we may assume that no curve in 
$\Gamma$ is mapped into a horizontal leaf and that $F^{-1}\left(\xi_{0}\right)$ is not in this cylinder. It follows that $F(\Gamma)$ has no locally rectifiable curve with respect to $D$. Now notice that $F(C)$ is a compact subset of $\partial G_{A} \backslash\left\{\xi_{0}\right\}$. By (5.1) the two metrics $d_{x}$ and $\rho_{b}$ are biLipschitz equivalent on $F(C)$. As $D$ and $\rho_{b}$ are biLipschitz equivalent, the metrics $d_{x}$ and $D$ are biLipschitz equivalent on $F(C)$. It follows that $F(\Gamma)$ has no locally rectifiable curve with respect to $d_{x}$. Hence $\operatorname{Mod}_{Q} F(\Gamma)=0$ in the metric measure space $\left(\partial G_{A}, d_{x}, \mu^{\prime}\right)$. Theorem 3.1 then implies that $\operatorname{Mod}_{Q} \Gamma=0$ in the metric measure space $\left(\partial G_{A}, d_{x}, \mu^{\prime}\right)$. On the other hand, $\operatorname{Mod}_{Q} \Gamma>0$ in the metric measure space $\left(\mathbb{R}^{n}, D, \mu\right)$ (see the proof of Theorem 3.2). Since $D$ and $d_{x}$ are biLipschitz equivalent on $C$, and $\mu$ and $\mu^{\prime}$ are also comparable on $C$, we have $\operatorname{Mod}_{Q} \Gamma>0$ in the metric measure space $\left(\partial G_{A}, d_{x}, \mu^{\prime}\right)$, a contradiction.

Next we prove that $F$ is biLipschitz with respect to the metric $D$. Since $F\left(\xi_{0}\right)=$ $\xi_{0}$, the map $F$ induces a map $F:\left(\partial G_{A} \backslash\left\{\xi_{0}\right\}, d_{x}\right) \rightarrow\left(\partial G_{A} \backslash\left\{\xi_{0}\right\}, d_{x}\right)$. Then (5.1) implies that $F:\left(\partial G_{A} \backslash\left\{\xi_{0}\right\}, \rho_{b}\right) \rightarrow\left(\partial G_{A} \backslash\left\{\xi_{0}\right\}, \rho_{b}\right)$ is quasisymmetric. Since $\rho_{b}$ and $D$ are biLipschitz equivalent, $F:\left(\partial G_{A} \backslash\left\{\xi_{0}\right\}, D\right) \rightarrow\left(\partial G_{A} \backslash\left\{\xi_{0}\right\}, D\right)$ is also quasisymmetric. Now the result follows from Theorem 4.1.

We note that because $G_{A}$ has sectional curvature $-\alpha_{r}^{2} \leq K \leq-\alpha_{1}^{2}, G_{A}$ is a proper geodesic $\delta$-hyperbolic space with $\delta$ depending only on $\alpha_{1}$.

Proof of Corollary 1.3. Suppose there is a quasiisometry $f: G_{A} \rightarrow G$ from $G_{A}$ to a finitely generated group $G$, where $G$ is equipped with a fixed word metric. Since $G_{A}$ is Gromov hyperbolic, it follows that $G$ is Gromov hyperbolic and $f$ induces a quasisymmetric map $\partial f: \partial G_{A} \rightarrow \partial G$. The left translation of $G$ on itself induces an action of $G$ on the Gromov boundary $\partial G$ by quasisymmetric maps. By conjugating this action with $\partial f$ we obtain an action of $G$ on $\partial G_{A}$ by quasisymmetric maps. By Theorem 1.1, this action has a global fixed point. It follows that the action of $G$ on $\partial G$ has a global fixed point. This can happen only when $G$ is virtually infinite cyclic, in which case the Gromov boundary $\partial G$ consists of only two points. This contradicts the fact that $\partial G_{A}$ is a sphere of dimension $n \geq 2$ (since $r \geq 2$ ).

The proofs of Corollaries 1.4 and 1.2 require some preparation.

Let $X$ be a proper geodesic $\delta$-hyperbolic space and $\xi_{1}, \xi_{2}, \xi_{3} \in X \cup \partial X$ be three distinct points. For any constant $C \geq 0$, a point $x \in X$ is called a $C$-quasi-center of the three points $\xi_{1}, \xi_{2}, \xi_{3}$ if for each $i=1,2,3$, there is a geodesic $\sigma_{i}$ joining $\xi_{i}$ and $\xi_{i+1}\left(\xi_{4}:=\xi_{1}\right)$ such that the distance from $x$ to $\sigma_{i}$ is at most $C$. For any $C \geq 0$, there is a constant $C^{\prime}$ that depends only on $\delta$ and $C$ such that the distance between any two $C$-quasi-centers of $\xi_{1}, \xi_{2}, \xi_{3}$ is at most $C^{\prime}$.

Notice that, if $X$ is a CAT $(-1)$ space, $\omega, \xi, \eta \in \partial X$ are three distinct points, $x \in X$ is a $C$-quasi-center of $\omega, \xi, \eta$ for some $C \geq 0$, and $b$ is a Busemann function associated to $\omega$, then $\left|(\xi \mid \eta)_{b}-b(x)\right| \leq C^{\prime}$ for some constant $C^{\prime}$ depending only on $C$. It follows that

$$
e^{-C^{\prime}} e^{-b(x)} \leq \rho_{b}(\xi, \eta) \leq e^{C^{\prime}} e^{-b(x)}
$$


Corollaries 1.2 and 1.4 follow from Theorem 1.1 and the following lemma. Recall that, the metric $\rho_{b}$ is biLipschitz equivalent to $D$, and by Theorem 1.1 , for any quasiisometry $f: G_{A} \rightarrow G_{A}$, the boundary map $\partial f$ fixes $\xi_{0}$ and restricts to a biLipschitz map of $\left(\partial G_{A} \backslash\left\{\xi_{0}\right\}, D\right)$, which we still denote by $\partial f$.

Let $M$ be a CAT(-1) space, $\xi_{0} \in \partial M$, and $x_{0} \in M$ a base point. Let $\gamma$ be the geodesic ray with $\gamma(0)=x_{0}$ and $\gamma(\infty)=\xi_{0}$, and $B_{\gamma}$ the Busemann function associated with $\gamma$. Let $\rho_{M}:=\rho_{B_{\gamma}}$ be the Hamenstädt metric associated with the Busemann function $B_{\gamma}$.

Let $N$ be another CAT(-1) space, and $f: M \rightarrow N$ an $\left(L_{0}, C_{0}\right)$-quasiisometry. For any $\xi \in \partial M$ and $x \in M$, we set $\xi^{\prime}=\partial f(\xi)$ and $x^{\prime}=f(x)$. Let $\gamma^{\prime}$ be the geodesic ray with $\gamma^{\prime}(0)=x_{0}^{\prime}$ and $\gamma^{\prime}(\infty)=\xi_{0}^{\prime}$, and $B_{\gamma^{\prime}}$ the Busemann function associated with $\gamma^{\prime}$. Let $\rho_{N}:=\rho_{\gamma^{\prime}}$ be the Hamenstädt metric associated with the Busemann function $B_{\gamma^{\prime}}$.

Lemma 5.1. Let $s>0$. Suppose that there exists a constant $C_{1} \geq 0$ such that every point $x \in M$ is a $C_{1}$-quasi-center of some distinct triple $\left(\xi_{0}, \xi, \eta\right)$ in $\partial M$. Then the following three conditions are equivalent:

(i) there is a constant $C \geq 0$ such that $\left|B_{\gamma^{\prime}}(f(x))-s \cdot B_{\gamma}(x)\right| \leq C$ for all $x \in M$;

(ii) the boundary map $\partial f:\left(\partial M \backslash\left\{\xi_{0}\right\}, \rho_{M}^{S}\right) \rightarrow\left(\partial N \backslash\left\{\xi_{0}^{\prime}\right\}, \rho_{N}\right)$ is biLipschitz;

(iii) there exists a constant $C \geq 0$ such that $s \cdot d(x, y)-C \leq d(f(x), f(y)) \leq$ $s \cdot d(x, y)+C$ for all $x, y \in M$.

Proof. (2) $\Longrightarrow$ (1). Suppose that the boundary map

$$
\partial f:\left(\partial M \backslash\left\{\xi_{0}\right\}, \rho_{M}^{s}\right) \rightarrow\left(\partial N \backslash\left\{\xi_{0}^{\prime}\right\}, \rho_{N}\right)
$$

is $L$-biLipschitz for some $L \geq 1$. Let $x \in M$. By assumption, $x$ is a $C_{1}$-quasi-center of some distinct triple $\left(\xi_{0}, \xi, \eta\right)$ in $\partial M$. By the property of Hamenstädt metric we have

$$
\frac{1}{C_{2}} e^{-B_{\gamma}(x)} \leq \rho_{M}(\xi, \eta) \leq C_{2} e^{-B_{\gamma}(x)}
$$

where $C_{2}$ depends only on $C_{1}$. Since $f: M \rightarrow N$ is an $\left(L_{0}, C_{0}\right)$-quasiisometry, the point $f(x)$ is a $C_{3}$-quasi-center of $\left(\xi_{0}^{\prime}, \xi^{\prime}, \eta^{\prime}\right)$ with $C_{3}=C_{3}\left(L_{0}, C_{0}, C_{1}\right)$. It follows that

$$
\frac{1}{C_{4}} e^{-B_{\gamma^{\prime}}(f(x))} \leq \rho_{N}\left(\xi^{\prime}, \eta^{\prime}\right) \leq C_{4} e^{-B_{\gamma^{\prime}}(f(x))},
$$

where $C_{4}$ depends only on $C_{3}$. Since $\partial f:\left(\partial M \backslash\left\{\xi_{0}\right\}, \rho_{M}^{S}\right) \rightarrow\left(\partial N \backslash\left\{\xi_{0}^{\prime}\right\}, \rho_{N}\right)$ is $L$-biLipschitz, (5.2) and (5.3) imply $\left|B_{\gamma^{\prime}}(f(x))-s \cdot B_{\gamma}(x)\right| \leq \ln \left(L C_{4} C_{2}^{s}\right)$.

$(1) \Longrightarrow(2)$. Suppose there is a constant $C$ such that $\left|B_{\gamma^{\prime}}(f(x))-s \cdot B_{\gamma}(x)\right| \leq$ $C$ for all $x \in M$. Let $\xi \neq \eta \in \partial M \backslash\left\{\xi_{0}\right\}$. Then the triple $\left(\xi_{0}, \xi, \eta\right)$ has a $C_{2^{-}}$ quasi-center $x \in M$, where $C_{2}$ is a universal constant. Since $f: M \rightarrow N$ is 
an $\left(L_{0}, C_{0}\right)$-quasiisometry, the point $f(x)$ is a $C_{3}$-quasi-center of $\left(\xi_{0}^{\prime}, \xi^{\prime}, \eta^{\prime}\right)$ with $C_{3}=C_{3}\left(L_{0}, C_{0}\right)$. Then we have

$$
\frac{1}{C_{4}} e^{-B_{\gamma}(x)} \leq \rho_{M}(\xi, \eta) \leq C_{4} e^{-B_{\gamma}(x)}
$$

and

$$
\frac{1}{C_{5}} e^{-B_{\gamma^{\prime}}(f(x))} \leq \rho_{N}\left(\xi^{\prime}, \eta^{\prime}\right) \leq C_{5} e^{-B_{\gamma^{\prime}}(f(x))},
$$

where $C_{4}$ depends only on $C_{2}$ and $C_{5}$ depends only on $C_{3}$. Now it follows from $\left|B_{\gamma^{\prime}}(f(x))-s \cdot B_{\gamma}(x)\right| \leq C$ that $\partial f:\left(\partial M \backslash\left\{\xi_{0}\right\}, \rho_{M}^{s}\right) \rightarrow\left(\partial N \backslash\left\{\xi_{0}^{\prime}\right\}, \rho_{N}\right)$ is $L-$ biLipschitz, where $L=C_{5} e^{C} C_{4}^{s}$.

(1) $\Longrightarrow$ (3). Suppose there is a constant $C \geq 0$ such that $\left|B_{\gamma^{\prime}}(f(x))-s \cdot B_{\gamma}(x)\right| \leq$ $C$ for all $x \in M$. Let $x, y \in M$. Let $p \in M$ be a $C_{2}$-quasi-center of $\left(x, y, \xi_{0}\right)$, where $C_{2}$ is a universal constant. Since $f: M \rightarrow N$ is an $\left(L_{0}, A_{0}\right)$-quasiisometry, $f(p)$ is a $C_{3}$-quasi-center of $\left(f(x), f(y), \xi_{0}^{\prime}\right)$ with $C_{3}=C_{3}\left(L_{0}, C_{0}, C_{2}\right)$. Then

$$
\begin{gathered}
\left|B_{\gamma}(x)-B_{\gamma}(p)-d(x, p)\right| \leq 2 C_{2}, \\
\left|B_{\gamma}(y)-B_{\gamma}(p)-d(y, p)\right| \leq 2 C_{2}, \\
\left|B_{\gamma^{\prime}}(f(y))-B_{\gamma^{\prime}}(f(p))-d(f(y), f(p))\right| \leq 2 C_{3}
\end{gathered}
$$

and

$$
\left|B_{\gamma^{\prime}}(f(x))-B_{\gamma^{\prime}}(f(p))-d(f(x), f(p))\right| \leq 2 C_{3} .
$$

Also notice that $|d(x, y)-d(p, x)-d(p, y)| \leq 2 C_{2}$ and

$$
|d(f(x), f(y))-d(f(p), f(x))-d(f(p), f(y))| \leq 2 C_{3} .
$$

These inequalities together with assumption (1) imply

$$
|d(f(x), f(y))-s \cdot d(x, y)| \leq 6 C_{3}+4 C+6 s C_{2} .
$$

(3) $\Longrightarrow(1)$ : Suppose (3) holds. Fix some $\xi \in \partial M \backslash\left\{\xi_{0}\right\}$. The image $f\left(\xi \xi_{0}\right)$ is an $\left(L_{0}, C_{0}\right)$-quasi-geodesic from $\xi^{\prime}$ to $\xi_{0}^{\prime}$. By the stability of quasi-geodesics, the Hausdorff distance $H D\left(f\left(\xi \xi_{0}\right), \xi^{\prime} \xi_{0}^{\prime}\right) \leq C_{2}$ for some constant $C_{2}=C_{2}\left(L_{0}, C_{0}\right)$. It follows from this and (3) that

$$
\left|B_{\gamma^{\prime}}(f(p))-B_{\gamma^{\prime}}(f(q))-s\left[B_{\gamma}(p)-B_{\gamma}(q)\right]\right| \leq C+4 C_{2}
$$

for all $p, q \in \xi \xi_{0}$. Fixing $q \in \xi \xi_{0}$ we obtain

$$
\left|B_{\gamma^{\prime}}(f(p))-s B_{\gamma}(p)\right| \leq C_{3}:=C+4 C_{2}+\left|B_{\gamma^{\prime}}(f(q))-s B_{\gamma}(q)\right|
$$

for all $p \in \xi \xi_{0}$. Now let $x \in M$ be arbitrary. Let $p \in \xi \xi_{0}$ be the projection of $x$ onto $\xi \xi_{0}$. Then $p$ is a $C_{4}$-quasi-center of $\left(x, \xi, \xi_{0}\right)$ for some universal constant $C_{4}$. It 
follows that $f(p)$ is a $C_{5}$-quasi-center of $\left(f(x), \xi^{\prime}, \xi_{0}^{\prime}\right)$ with $C_{5}=C_{5}\left(L_{0}, C_{0}, C_{4}\right)$. Hence

$$
\left|B_{\gamma}(x)-B_{\gamma}(p)-d(x, p)\right| \leq 2 C_{4}
$$

and

$$
\left.\mid B_{\gamma^{\prime}}(f(x))-B_{\gamma^{\prime}}(f(p))-d(f(x)), f(p)\right) \mid \leq 2 C_{5} .
$$

Now (5.4), (5.5), (5.6) and assumption (3) imply

$$
\left|B_{\gamma^{\prime}}(f(x))-s B_{\gamma}(x)\right| \leq C+2 C_{5}+C_{3}+2 s C_{4}
$$

for all $x \in M$.

\section{References}

[B] Z. Balogh, Hausdorff dimension distribution of quasiconformal mappings on the Heisenberg group. J. Anal. Math. 83 (2001), 289-312. Zbl 0983.30007 MR 1828495

[BK] M. Bonk and B. Kleiner, Rigidity for quasimobius group actions. J. Differential Geom. 61 (2002), no. 1, 81-106. Zbl 1044.37015 MR 1949785

[Bo] M. Bourdon, Structure conforme au bord et flot geodesique d'un CAT(-1)-espace. Enseign. Math. (2) 41 (1995), 63-102. Zbl 0871.58069 MR 1341941

[BP] M. Bourdon and H. Pajot, Rigidity of quasi-isometries for some hyperbolic buildings. Comment. Math. Helv. 75 (2000), no. 4, 701-736. Zbl 0976.30011 MR 1789183

[D] T. Dymarz, Large scale geometry of certain solvable groups. Geom. Funct. Anal. 19 (2010), no. 6, 1650-1687. Zbl 1211.20037 MR 2594617

[EFW1] A. Eskin, D. Fisher and K. Whyte, Coarse differentiation of quasi-isometries I: spaces not quasi-isometric to Cayley graphs. Preprint, arXiv:math/0607207v2 [math.GR].

[EFW2] A. Eskin, D. Fisher and K. Whyte, Coarse differentiation of quasi-isometries II: Rigidity for Sol and Lamplighter groups. Preprint, arXiv:0706.0940v1 [math.GR].

[FM1] B. Farb and L. Mosher, On the asymptotic geometry of abelian-by-cyclic groups. Acta Math. 184 (2000), no. 2, 145-202. Zbl 0982.20026 MR 1768110

[FM2] B. Farb and L. Mosher, A rigidity theorem for the solvable Baumslag-Solitar groups. Invent. Math. 131 (1998), no. 2, 419-451. Zbl 0937.22003 MR 1608595

[FM3] B. Farb and L. Mosher, Quasi-isometric rigidity for the solvable Baumslag-Solitar groups. II. Invent. Math. 137 (1999), no. 3, 613-649. Zbl 0931.20035 MR 1709862

[FS] T. Foertsch and V. Schroeder, Hyperbolicity, CAT(-1)-spaces and the Ptolemy inequality. Math. Ann. 350 (2011), no. 2, 339-356. Zbl 1219.53042 MR 2794913

[GV] F. Gehring and J. Vaisala, Hausdorff dimension and quasiconformal mappings. J. London Math. Soc. (2) 6 (1973), 504-512. Zbl 0258.30020 MR 0324028

[H] U. Hamenstädt, A new description of the Bowen-Margulis measure. Ergodic Theory Dynam. Sysytems 9 (1989), no. 3, 455-464. Zbl 0722.58029 MR 1016663 
Vol. 87 (2012) A rigidity property of some negatively curved solvable Lie groups

[HP] S. Hersonsky and F. Paulin, On the rigidity of discrete isometry groups of negatively curved spaces. Comment. Math. Helv. 72 (1997), 349-388. Zbl 0908.57009 MR 1476054

[K] B. Kleiner, Unpublished notes. 2001.

[P] P. Pansu, Metriques de Carnot-Caratheodory et quasiisometries des espaces symetriques de rang un. Ann. of Math. (2) 129 (1989), no. 1, 1-60. Zbl 0678.53042 MR 0979599

[Pe] I. Peng, Coarse differentiation and quasi-isometries of a class of solvable Lie groups I. http://front.math.ucdavis.edu/0802.2596

[T1] J. Tyson, Quasiconformality and quasisymmetry in metric measure spaces. Ann. Acad. Sci. Fenn. Math. 23 (1998), no. 2, 525-548. Zbl 0910.30022 MR 1642158

[T2] J. Tyson, Metric and geometric quasiconformality in Ahlfors regular Loewner spaces. Conform. Geom. Dyn. 5 (2001), 21-73. Zbl 0981.30015 MR 1872156

[V1] J. Väisälä, Lectures on n-dimensional quasiconformal mappings. Lecture Notes in Math. 229, Springer-Verlag Berlin 1971. Zbl 0221.30031 MR 0454009

[V2] J. Väisälä, The free quasiworld. Freely quasiconformal and related maps in Banach spaces. In Quasiconformal geometry and dynamics Banach Center Publ. 48, Polish Academy of Sciences, Institute of Mathematics, Warsaw 1999, 55-118. Zbl 0934.30018 MR 1709974

[X] X. Xie, Quasi-isometric rigidity of Fuchsian buildings. Topology 45 (2006), no. 1, 101-169. Zbl 1083.51008 MR 2170496

Received September 22, 2009

Nageswari Shanmugalingam, Department of Mathematical Sciences, P.O.Box 210025,

University of Cincinnati, Cincinnati, OH 45221-0025, U.S.A.

E-mail: shanmun@uc.edu

Xiangdong Xie, Department of Mathematics and Statistics, Bowling Green State University,

Bowling Green, OH 43403-0221, U.S.A.

E-mail: xiex@bgsu.edu 